\section{Two catheter technique in neonatal peritoneal dialysis}

Sir,

An occasional need for short term peritoneal dialysis arises in the management of small neonates, in both neonatal intensive care and after cardiac surgery. The conventional technique using a single catheter can be problematic and it is sometimes difficult to maintain an accurate fluid balance due to the small volumes used, leakage around the catheter, and erratic return volumes. Furthermore, respiratory embarrassment from splinting of the diaphragm by the intraperitoneal fluid may occur.

An alternative technique using two catheters is described. The catheters (Vygon neonatal chest drains with extra holes cut in the walls) were inserted just lateral of the midline and below the umbilicus, with their tips directed to each iliac fossa. A continuous flow of warm dialysis fluid was maintained using a paediatric volumetric infusion pump attached to one catheter, the other draining freely into an accurate measurement system.

The technique was used for periods of 36 hours to 4 days in three neonates weighing $800 \mathrm{~g}, 1200 \mathrm{~g}$, and $3100 \mathrm{~g}$. Although all three patients died of their primary problems (severe hypoxia in two and perforated necrotizing enterocolitis in the third), the dialysis was technically successful in correcting fluid and biochemical imbalance and proved simple for the nursing and medical staff to manage, overcoming the problems described with single catheters. A cross flow of $100 \mathrm{ml} / \mathrm{kg} / \mathrm{hour}$ was found to be adequate. There is a further theoretical advance to cross flow, the absence of frequently manipulated three way taps and a high volume continuous flow ought to reduce the risk of infection.

J G Davies Coventry Maternity Hospital, Clifford Bridge Road, Walsgrave,

Coventry CV2 $2 D X$

\section{A syndrome in the rat resembling pink disease}

Sir,

In their paper of 55 years ago quoted recently in the Archives $^{1}$ about a syndrome resembling pink disease, Drs Findlay and Stern showed clearly that the illness they induced in the rats was due to a factor in the diet. They postulated deficiency of a vitamin. They were of course correct as they were describing biotin deficiency. The symptoms, namely the rash (particularly at the mucocutaneous junctions), alopecia, and neurological involvement are all similar to those of biotin deficiency in man. The odour was probably due to the organic acids that are formed because of the deficient carboxylase activity and the infection to secondary immunodeficiency. The neuropathological findings were not specific (Dr Brian Harding - personal communication).

It is difficult to make adult rats deficient on a biotin free diet and it is necessary to prevent coprophagy: young rats are more easily rendered biotin deficient. The addition of egg white accelerates the process because it contains avidin, a protein which binds biotin so that it is no longer available to the animal. Health was restored by giving foods with a high biotin content such as raw liver.

Reference
${ }^{1}$ Stern RO. Fifty five years ago. A syndrome in the rat
resembling pink disease in man. Arch Dis Child 1984;59:179.
(originally published Arch Dis Child $1929 ; 4: 1-11$.
J V LEONARD
Institute of Child Health,
30 Guilford Street,
London WCIN $1 E H$

\section{Infections in a neonatal intensive care unit}

Sir,

We read with great interest the article on the study of chlamydial, mycoplasmal, and viral infections by Rudd and Carrington. ${ }^{1}$ In our experience at the Southern General Hospital, Glasgow we feel the incidence of neonatal chlamydial infection is probably more than $1.4 \%$. In 1983 , out of 2622 neonates, 50 cases of chlamydial conjunctivitis were identified $(>1.9 \%)$. There were probably many more cases of chlamydial infection treated by general practitioners or in other hospitals as many of our mothers were discharged 48 hours after delivery.

Most of the babies with chlamydial conjunctivitis were treated with topical tetracycline with good response. Not a single infant developed pneumonia.

In our unit the most common cause of ophthalmia neonatorum was found to be Chlamydia trachomatis. Chlamydia was also identified in some cases of ophthalmia where the infant was delivered by caesarean section.

We feel there is a need to identify women infected with Chlamydia trachomatis around the time of delivery so that their babies can be given prophylactic systemic erythromycin, and conjunctivitis and pneumonia can thereby be prevented.

\section{Reference}

1 Rudd PT, Carrington D. A prospective study of chlamydial, mycoplasmal, and viral infections in a neonatal intensive care unit. Arch Dis Child 1984;59:120-5.

R A GARG

Paediatric Unit, Stobhill General Hospital, Glasgow 